\title{
Discectomy with Subsequent Free Fat Flap Insertion in Disc Perforation Therapy of Temporomandibular Joint. Assessment of Results 24 Months after Operation
}

\section{Vladimír Machoň, Juhi Vir, Jitka Levorová, Michal Beňo, Dušan Hirjak, René Foltán}

Department of Dental Medicine, First Faculty of Medicine, Charles University and General University Hospital in Prague, Prague, Czech Republic

Received December 16, 2019; Accepted May 28, 2020.

Key words: Discectomy - Disc perforation - Free fat flap - Temporomandibular joint

Abstract: Discectomy with replacement of disc is one possibility for treating disc perforation where conservative, mini-invasive therapy and arthroscopy has had no effect. Allogenic or autologous materials are used to replace the disc. The authors assess the use of a free fat flap (FFF) in 19 patients who in 2015-2016 underwent a unilateral discectomy with disc replacement. In the retrospective 24-month follow-up study a total of 16 patients (84\%) were free of difficulties. 24 months after the operation mouth opening was on average $39.3 \mathrm{~mm}$, pain (VAS - visual analog scale (0-10) was assessed on average at 0.3). Crepitus was present after 24 months in $37.5 \%$ of patients ( 6 patients). An assessment of changes in joint structures on cone beam computed tomography (CBCT) for these patients 24 months after the operation showed the progression of flattening of the joint head, in one case unevenness of the joint head. In 3 cases (16\%) there was a recurrence of the state within 24 months - in all cases with clinical manifestations of pain and limited mobility, for these patients on the CBCT significant unevennesses of the joint head, subchondral cysts were noted. The authors find discectomy with use of FFF to be an effective method of treatment with a minimum of complications. However, one should take into account the relatively short time of monitoring after the operation ( 2 years) and limited number of patients in the cohort (19 patients).

Mailing Address: Vladimír Machoň, MD., Department of Dental Medicine, First Faculty of Medicine, Charles University and General University Hospital in Prague, U Nemocnice 2, 12000 Prague 2, Czech Republic; e-mail: machonv@seznam.cz 


\section{Introduction}

Perforation of the disc of the temporomandibular joint occurs as a result of degenerative changes, and it can be divided into central (localised in the area of the actual disc) and peripheral (which occurs in the transitional zone, at the insertion point of the retrodiscal tissue on the disc). Disc perforations are accompanied by many clinical signs (crepitus during jaw movement, pain, limitation of jaw mobility). They can also be completely asymptomatic. Asymptomatic perforation is not an indication for therapy - objective and subjective difficulties of the patient constitute an indication. Diagnosis of perforation relies on magnetic resonance imaging (MRI), but the most accurate diagnostic method for confirming perforation is arthroscopy (ASC) (Laskin et al., 2006; Machon et al., 2017). An arthroscopic lavage is a successful treatment method, as confirmed by the authors in their previous work (Machon et al., 2017). If the arthroscopy has no effect, in the case of disc perforation the disc is removed and possibly replaced (but only if at the same time only slight changes in the joint head and fossa are present). If as well as disc perforation there are extensive degenerative changes to the condyle or fossa (erosion, subchondral cysts), in addition to a discectomy it is possible to carry out at the same time a resection of the condyle with subsequent total joint replacement (Mercuri, 2008; Machoň, 2017).

The specified materials (allogenic or autologous) for replacement of the disc are: silicone sheets, autogenous dermal graft, dermis-fat graft, auricular cartilage graft, temporoparietal fascia flap, and temporalis myofascial flap, free fat flap) (Dimitroulis, 2011; DeMerle et al., 2017).

The aim of the authors' work is to evaluate the effect of a discectomy with subsequent insertion of free fat flap into the joint cavity in a 2-year retrospective study.

\section{Material and Methods}

The cohort consisted of 19 patients (in all cases they were women, the average age was 53.7, the ages ranged from 20 to 71). The cohort included only patients with unilateral disc perforation who in the years 2015-2016 had undergone arthroscopic lavage following previous ineffective conservative therapy. Patients with a bilateral

\section{Table 1 - Symptoms of patients indicated for discectomy with subsequent use of FFF}

\begin{tabular}{lr}
\hline Symptoms \\
\hline Limited jaw mobility $(\mathrm{MIO}<30 \mathrm{~mm})$ and pain (VAS $>2)$ & 11 patients $(58 \%)$ \\
Limited jaw mobility only $(\mathrm{MIO}<30 \mathrm{~mm})$ & 4 patients $(21 \%)$ \\
Pain only $(\mathrm{VAS}>2)$ & 4 patients $(21 \%)$ \\
\hline
\end{tabular}

FFF - free fat flap; MIO - maximal interincisal mouth opening; VAS - visual analog scale 
affliction were not included in the cohort, nor were patients with autoimmune or endocrine diseases.

The indication for discectomy with subsequent insertion of FFF (free fat flap) were ongoing difficulties (pain, limitation of jaw mobility) for at least 3-6 months following a preceding arthroscopy. The clinical symptoms before extirpation of the disc were pain and limitation of jaw mobility in 11 patients (58\%), limited jaw mobility only in 4 patients (21\%), and pain only in 4 patients (21\%) (Table 1).

The presence of noise phenomena alone (crepitus) did not constitute an indication for the operation.

Another criterion for indication of discectomy with use of FFF was the presence of only slight changes of joint structure in the sense of flattening of the joint head. Patients where joint head erosions were present, where there were subchondral cysts and changes in the fossa, were dealt with by resection of the joint structures with subsequent reconstruction by total joint replacement.

After the operation the patients underwent regular check-ups at 2 weeks, 1, 3, 6, 12 and 24 months. During examinations there was an assessment of maximum mouth opening (MIO), pain (VAS - visual analog scale 0-10), and presence of noise phenomena and symmetry of mouth opening. For this work there was an evaluation of the results of examinations before the surgery and then 3,12 and 24 months after the surgery, and the value for mouth opening ( $\mathrm{MIO}$ - maximal interincisal mouth opening) and pain (VAS) was used for patients where there was no recurrence of the state during the monitored period of 24 months. For all patients undergoing this surgery cone beam computed tomography (CBCT) was carried out before and 24 months after the surgery.

Check-up CBCT was performed for all patients where there was a recurrence of pain and limitation of mobility. In the case of progression of the degenerative changes the state was dealt with by resection of the joint process and subsequent reconstruction of the joint using a custom made total joint replacement (TMJ Concepts, USA).

\section{Performance of surgery}

The surgery was performed under general anaesthetic (with nasotracheal intubation). In all cases the endaural approach was selected, then the area of the joint capsule was penetrated via deep subfascial approach and opened with a "T" incision in the lower and upper joint cavity. There followed an eminectomy and disc extirpation including retrodiscal tissue, lavage of joint cavities. Then the free fat flap (FFF) was taken from the umbilical region and inserted in the joint cavity so that it filled it entirely (Figures 1-4). Finally, the wounds were sutured (Machoň, 2017).

Postoperative regime

Postoperative regime consisted of limiting jaw mobility for 3 days, soft foods. From the $4^{\text {th }}$ day after the surgery the patient started to open the mouth again as part of 


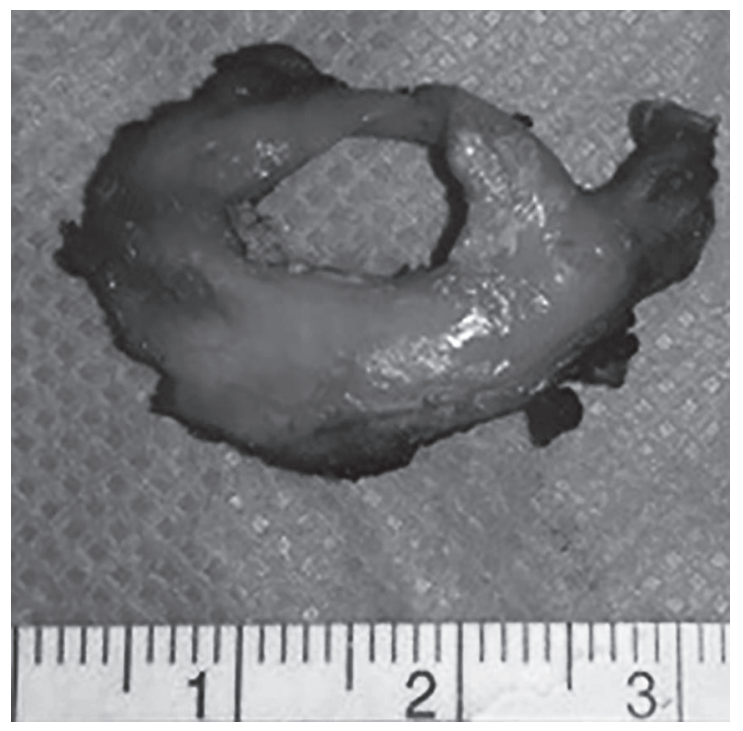

Figure 1 - The perforation of the disc, disc after discectomy.

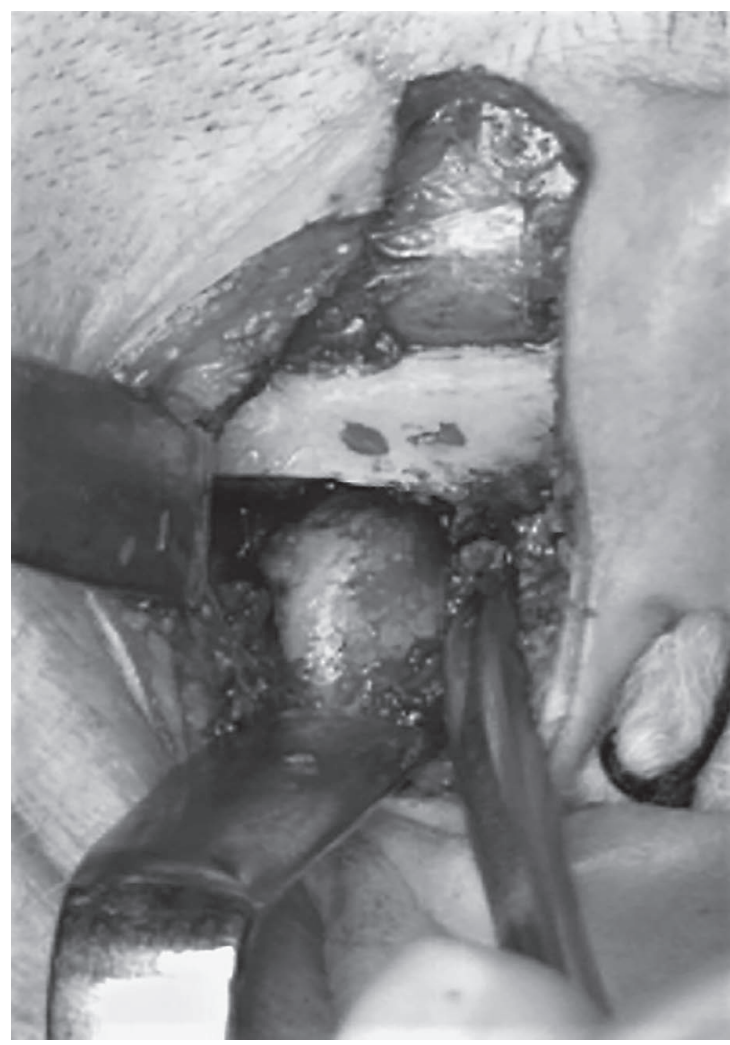

Figure 2 - Temporomandibular joint after discectomy.

Free Fat Flap in Disc Perforation Therapy 
100) Prague Medical Report / Vol. 121 (2020) No. 2, p. 96-106

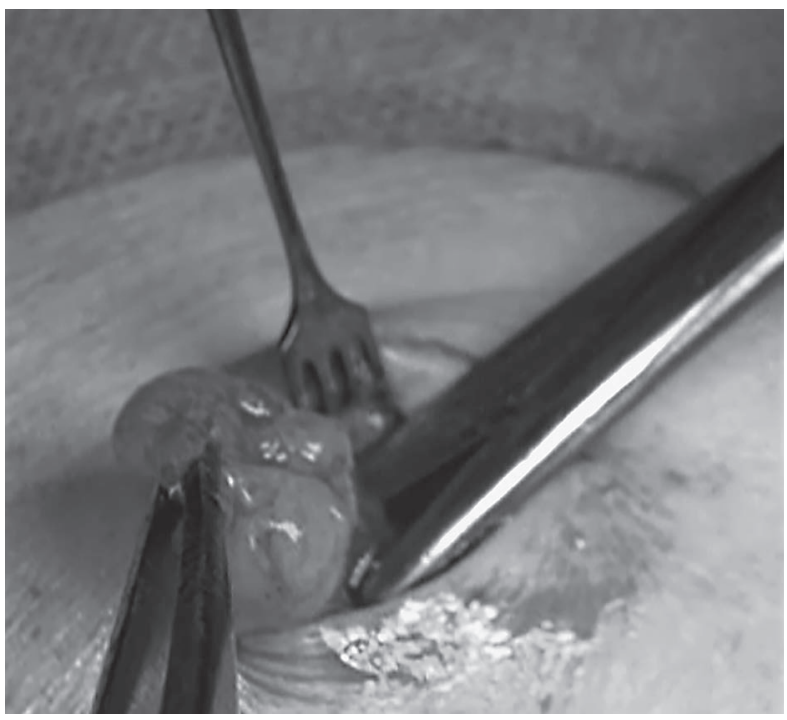

Figure 3 - Preparation of free fat flap from the umbilical region.

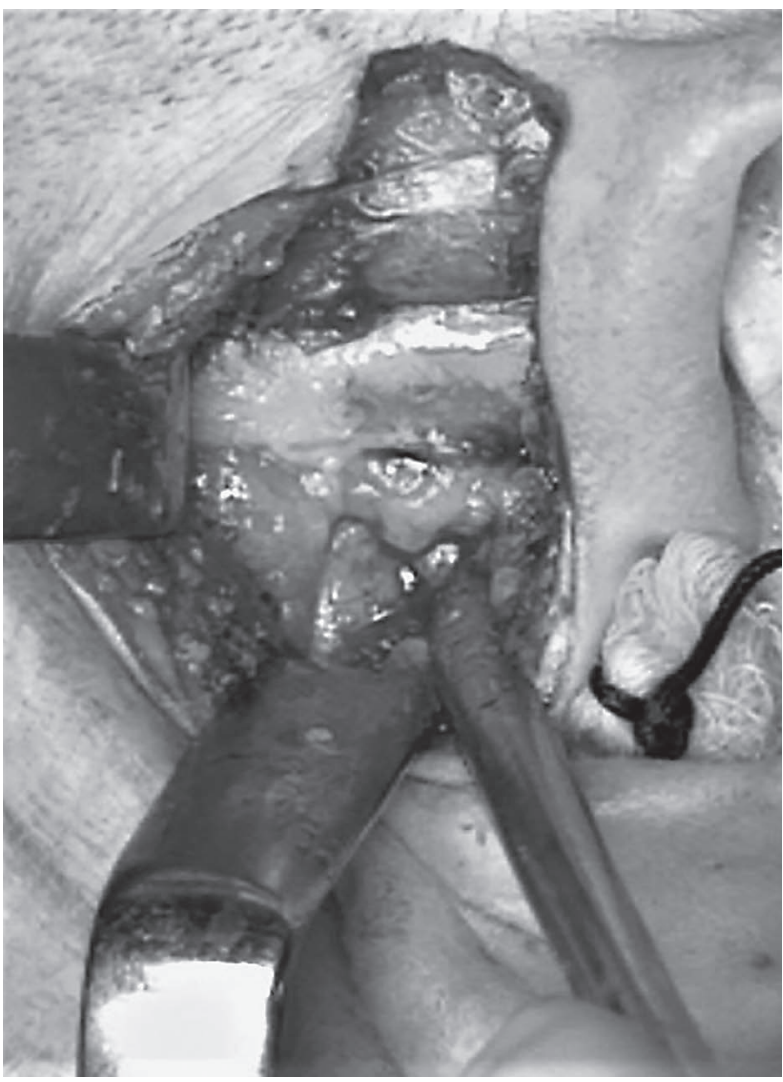

Figure 4 - The joint cavity with free fat flap.

Machoň V.; Vir J.; Levorová J.; Beňo M.; Hirjak D.; Foltán R. 
physiotherapy (gradually, up to the pain threshold). An occlusal splint (1.5 mm thick, on lower teeth) was recommended for at least 3 months after the operation, for use 24 hours a day if possible. It was recommended that the occlusal splint be worn regularly at night for 3 months after this.

If hypofunction of the facial nerve was noted during the post-operative period, rehabilitation of the nerve function was initiated immediately on the first day after the operation (Machoň, 2017).

Antibiotics were prescribed for the first week after surgery (Co-Amoxiclav, or for patients who were allergic to penicillin - Clindamycin).

\section{Results}

Out of the 19 patients who underwent discectomy with FFF insertion, the operation was ineffective for 3 patients over the monitoring period of 2 years. In 1 patient the difficulties recurred during the first 12 months after the surgery, and in two others 13-24 months after the surgery. In all three patients according to the CBCT there was evident progression in the degenerative changes of joint structures - in all three cases the state was dealt with by a resection of the joint process with subsequent reconstruction of joint.

In the case of patients where there had been no recurrence of the clinical difficulties, 24 months after the operation progression in the flattening of the joint head was observed on the CBCT (in 15 patients), and in 1 case the presence of erosion changes of the joint head.

In 16 patients (84\%) the state was stable 24 months after the surgery (opening of mouth more than $30 \mathrm{~mm}$, pain subjectively evaluated at VAS $0-2)$. In these patients

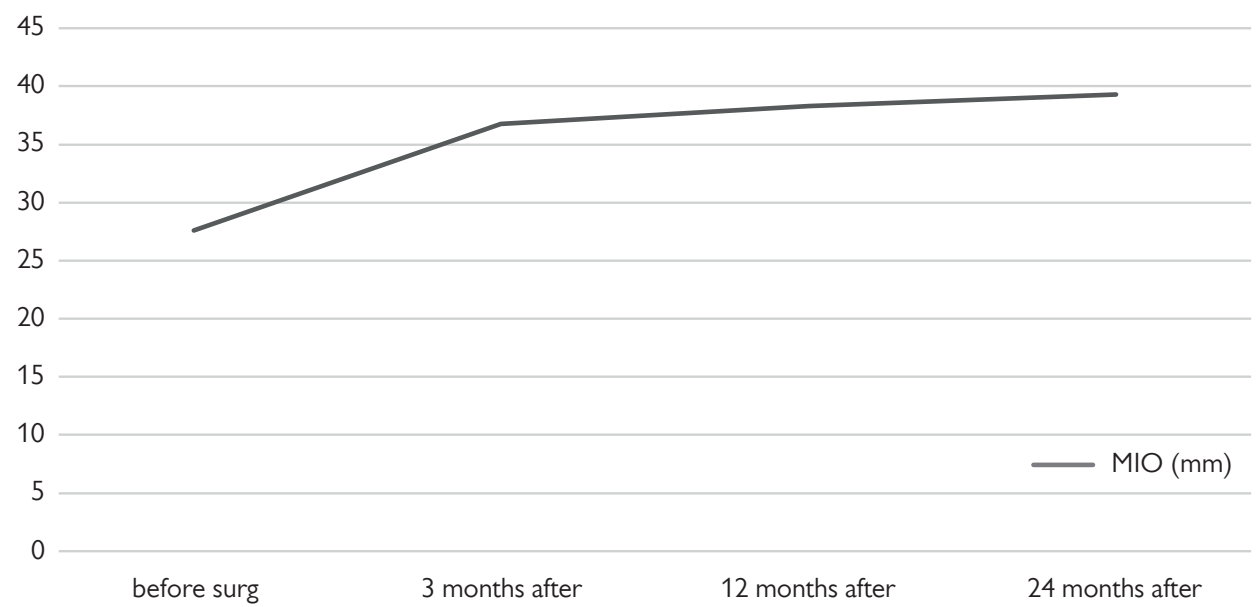

Figure 5 - Evaluation of opening of the mouth in patients where there was no recurrence of the state (16 patients). 


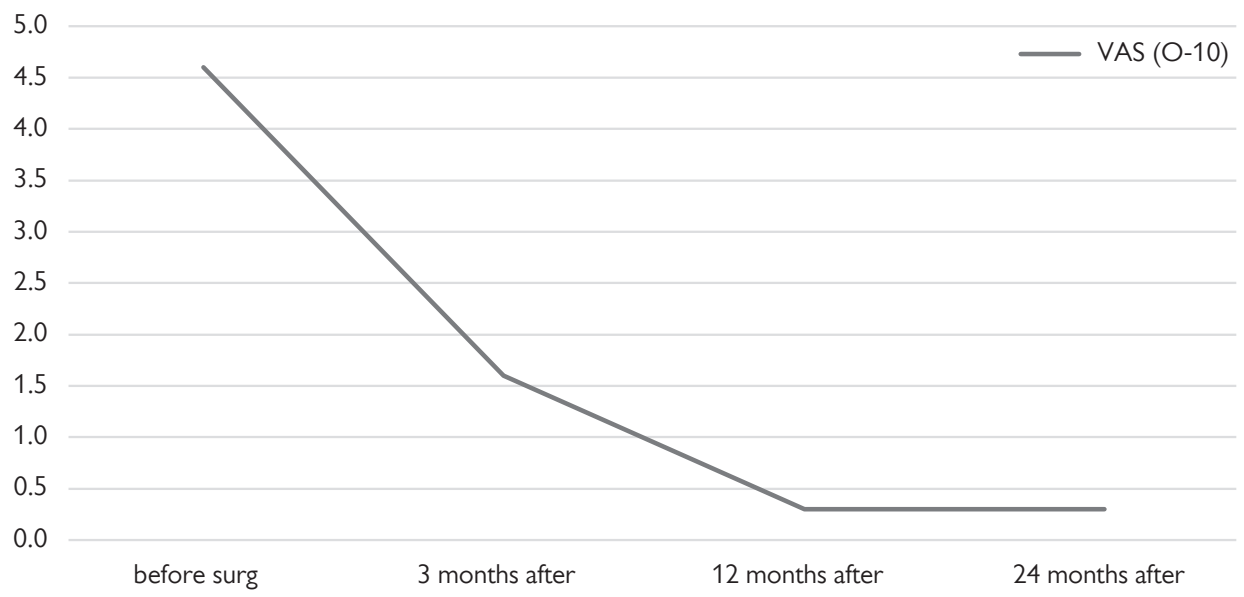

Figure 6 - Evaluation of pain (VAS 0-10) in patients where there was no recurrence of the state (16 patients).

the average value of MIO before the operation was $27.6 \mathrm{~mm}, 3$ months after the operation $36.8 \mathrm{~mm}, 12$ months after the operation $38.3 \mathrm{~mm}$, and 24 months after the operation $39.3 \mathrm{~mm}$ (Figure 5).

In all these patients before the operation a deviation had been observed during the opening of the mouth - with deflection of jaw on the afflicted side, 12 and 24 months after the operation symmetrical movement without deviation was achieved in 6 patients (37.5\%). In the other patients the movement deviation remained unchanged (10 patients, 62.5\%).

The average pain value was given by patients as 4.6 (VAS) before the operation. Three months after the operation the average pain value was 1.6. The average pain value was 0.3 (Figure 6) one and two years after the operation.

In 8 patients (out of 16 patients with effective therapy; 50\%), there was a noise phenomenon present before the operation in the form of crepitus, after the operation (3, 12 and 24 months) the noise phenomenon was present in 6 patients (37.5\%).

In all cases the operation was performed by a single surgeon, the average operation time was 50 minutes. Post-operative complications were noted in 1 patient (temporary paralysis of ramus temporalis of facial nerve - there was a correction over a period of three weeks following the operation).

In all cases the patients evaluated the healing of scars from the surgical wounds as satisfactory, without complications, in one patient dehiscence of the wound in the umbilical region was observed (but this occurred on the $10^{\text {th }}$ day after surgery, after the patient had fallen).

No other complications were observed. 


\section{Discussion}

Discectomy is a therapeutic method in patients with disk perforation where preceding therapy (conservation treatment, mini-invasive therapy and arthroscopy) have failed. Persistent pain or mobility block is an indication for a discectomy (Miloro and Henriksen, 2010; Machoň, 2017; Miloro et al., 2017). But the removal of the disc results in direct contact between joint structures and a change of mechanical load (Silver, 1984). This leads to changes in the joint head, in particular to increased sclerotization of the subchondral bones, flattening of the head. Agerberg and Lundberg (1971) described an initial "destructive" phase during the first 6 to 9 months characterized by cortical breakdown. This was followed by a "productive" phase with reconstitution of the cortical surface and expression of altered condylar morphology by 2 years. These morphological changes are not evaluated as progression of degenerative changes, but they represent functional adaptation changes (Silver, 1984; Eriksson and Westesson, 1992; Dimitroulis, 2011). In the work of the authors change in the shape of the joint head were observed over the 2 years following the operation in all patients. In 15 cases progression of flattening of the joint head, in 4 cases erosion - unevenness of the joint head was noted. But clinical symptoms in the sense of post-operative pain and limited mobility were present in only 3 patients - in all cases with unevennesses, erosion of joint head.

Other complications of discectomy are the formation of post-operative adhesions (in cavity created after removal of disc), the formation of ankylotic changes, heterotopic bone formation (Dolwick and Aufdemorte, 1985; Dimitroulis, 2011; Shen et al., 2015). In the 24-month follow-up study the authors did not observe any signs of the formation of heterotopic bone formation, ankylotic changes.

The use of interpositional material between the condyle and cranial base limited the progress of remodelling changes, the formation of adhesions and ankylotic changes (Dolwick and Aufdemorte, 1985). The materials used for disc replacement may be allogenic or autologous (silicone sheets, autogenous dermal graft, dermis-fat graft, auricular cartilage graft, temporoparietal fascia flap, and temporalis myofascial flap, free fat flap) (Dimitroulis, 2011; DeMerle et al., 2017). One question is the ideal interpositional material.

Dimitroulis (2011) presented the criteria for use of interpositional material:

- adequate bulk (fills up the whole joint cavity)

- good handling properties (remains intact during transfer, easy to sculpture during operation, can be easily moulded to fit the entire joint space)

- easy to procure (simple and quick operation; minimal donor morbidity; hidden scar)

- abundantly available (excess tissue available than what is required; can be harvested from multiple sites)

- survives the intra-joint environment (able to adapt to the functional joint demands; does not fragment or degenerate over time) 
- facilitates normal joint function (reduces joint noises; permits full range of joint motion; allows pain free joint function)

- prevents bone formation and joint ankylosis (acts as an effective barrier to calcification; eliminates heterotopic bone formation)

- protects condyle from severe remodelling (provides a buffer between the articular surfaces; counteracts the process responsible for condylar degeneration placed)

- long-term safety - data $>2$ years available

As evident from the literature, there is no ideal material for disc replacement.

Use of allogenic materials is associated with foreign body reactions, potential cross-infection and unpredictable resorption (Dolwick and Aufdemorte, 1985; Dimitroulis, 2011).

In the case of autologous materials, the $2^{\text {nd }}$ place of collection and thus the aesthetic handicap in the form of a scar is a complication. In the work of the authors the surgical scars in the umbilical region were assessed in 18 cases as satisfactory, in one case there occurred dehiscence of the surgical scar - as a result of the patient falling 10 days after the operation. Other complications of autologous materials are fragmentation of material (when FFF is used), resorption of volume of graft (use of FFF, ear cartilage), epidermoid cyst formation (use of full thickness skin), fibrosis and trismus (use of temporalis muscle), myofascial pain (use of temporalis muscle) (Feinberg and Larsen, 1989; Dimitroulis et al., 2004; Dimitroulis, 2005, 2011; DeMerle et al., 2017).

In the work of the authors there is an evaluation of the use of the free fat flap as interpositional material.

The first description of use of free fat flap as interpositional material is from 1914, where Murphy used it during the treatment of ankylosis (Murphy, 1914). Wolford and Karras (1997) recommend the use of FFF as prevention of heterotopic bone formation and reankylosis in patients who have undergone a total temporomandibular joint replacement. FFF filling the joint cavity or cavity following the resection of joint structures acts as an effective haemostatic in the joint cavity, it prevents adhesions forming (Shen et al., 2015).

The disadvantage of the free fat flap is its fragmentation, which reduces its effect as an interpositional material (Dimitroulis et al., 2004). The fragmentation of the fat flap is reduced by its combination in a joint dermal-fat graft (Dimitroulis et al., 2004; Dimitroulis, 2005, 2011). Another disadvantage of FFF is the reduction in the volume, its resorption. The reduction in the volume of the FFF may be by $1 / 3$ of the original volume during the first year after the operation (Peer, 1950; Kanamori et al., 2001; Dimitroulis, 2011).

Shen et al. (2015) evaluated the reduction in volume of inserted fat on MRI in 267 patients who underwent disc repositioning and where FFF was inserted as prevention against the formation of adhesions. During an examination after 
1-3 months the volume of fat had reduced to $57.82 \%$, and after 24 months to $48.44 \%$. Tekin et al. (2014) state the presence of FFF on CT (computed tomography) even 8 years after the insertion of a fat flap, this involved the case histories of patients where unsuitable alloplastic joint replacements were removed. The use of the bucal pedicle flap (BPF) may influence resorption. BPF minimises another disadvantage of FFF - the necessary place of collection, $2^{\text {nd }}$ surgical wound in the umbilical region (Rattan, 2006). Gaba et al. (2012) used BPF as an interpositional material after resection of ankylosis, during a check-up one year after the operation the fat flap was visualised on MRI in $65 \%$ of patients.

With regard to the clinical effect of discectomy with subsequent use of FFF: 24 months after the operation $84 \%$ of patients (16 patients) had no clinical difficulties (opening of the mouth on average $39.3 \mathrm{~mm}$ ), pain was evaluated at an average of 0.3 . Crepitus was present after 24 months in $37.5 \%$ of patients (6 patients). These results correspond with other authors and the use of other interpositional materials (Dimitroulis et al., 2004, 2010; Candirli et al., 2012; DeMerle et al., 2017; Muñoz-Guerra et al., 2018), or the results of patients who underwent only discectomy without replacement (Eriksson and Westesson, 1985; Miloro et al., 2017).

\section{Conclusion}

Discectomy with insertion of free fat flap is one possibility for dealing with symptomatic disk perforations where there are also only minimal changes in joint structures. The previous failure of conservative and mini-invasive therapies remains important in the indication criteria.

For the aforementioned criteria the authors find this treatment effective, with a minimum of complications. However, one should take into account the relatively short time of monitoring after the operation ( 2 years) and limited number of patients in the cohort (19 patients).

\section{References}

Agerberg, G., Lundberg, M. (1971) Changes in the temporomandibular joint after surgical treatment. A radiological follow-up study. Oral Surg. Oral Med. Oral Pathol. 32, 865-875.

Candirli, C., Esen, A., Taskesen, F., Celik, S., Cakir, B. (2012) Clinicoradiological evaluation of dermis-fat grafts after temporomandibular joint discectomy: a retrospective study. Natl. J. Maxillofac. Surg. 3(1), $42-46$.

DeMerle, M., Nafiu, O., Aronovich, S. (2017) Temporomandibular joint discectomy with abdominal fat graft versus temporalis myofascial flap: a comparative study. J. Oral Maxillofac. Surg. 75, 1137-1143.

Dimitroulis, G. (2005) The use of dermis grafts after discectomy for internal derangement of the temporomandibular joint. J. Oral Maxillofac. Surg. 63(2), 173-178.

Dimitroulis, G. (2011) A critical review of interpositional grafts following temporomandibular joint discectomy with an overview of the dermis-fat graft. Int. J. Oral Maxillofac. Surg. 40, 561-568.

Dimitroulis, G., Lee, D. K., Dolwick, M. F. (2004) Autogenous ear cartilage grafts for treatment of advanced temporomandibular joint disease. Ann. Roy. Australas. Coll. Dent. Surg. 17, 87-92. 
Dimitroulis, G., McCullough, M., Morrison, W. (2010) Quality-of-life survey comparing patients before and after discectomy of the temporomandibular joint. J. Oral Maxillofac. Surg. 68, 101-106.

Dolwick, M. F., Aufdemorte, T. B. (1985) Silicone induced foreign body reaction and lymphadenopathy after temporomandibular joint arthroplasty. Oral Surg. Oral Med. Oral Pathol. 59, 449-452.

Eriksson, L., Westesson, P. L. (1985) Long-term evaluation of menisectomy of the temporomandibular joint. J. Oral Maxillofac. Surg. 43, 263-269.

Eriksson, L., Westesson, P. L. (1992) Temporomandibular joint discectomy. No positive effect of temporary silicone implant in 5-year follow-up. Oral Surg. Oral Med. Oral Pathol. 74, 259-272.

Feinberg, D. E., Larsen, P. L. (1989) The use of a pedicled temporalis muscle-pericranial flap for replacement of the TMJ disc: preliminary report. J. Oral Maxillofac. Surg. 47, 142-146.

Gaba, S., Sharma, R. K., Rattan, V., Khandelwal, N. (2012) The long-term fate of pedicled buccal pad fat used for interpositional arthroplasty in TMJ ankylosis. J. Plast. Reconstr. Aesthet. Surg. 65, 1468-1473.

Kanamori, M., Kawaguchi, Y., Ohmori, K., Kimura, T., Tsuji, H., Matsui, H. (2001) The fate of autogenous free-fat grafts after posterior lumbar surgery: part 2. Magnetic resonance imaging and histologic studies in repeated surgery cases. Spine (Phila. Pa. 1976) 26, 2264-2270.

Laskin, D. M., Greene, C. S., Hylander, W. L. (2006) TMDs: An Evidence-based Approach to Diagnosis and Treatment. Quintessence Publ., Chicago.

Machoň, V. (2017) Manual of TMJ Surgery; The Prague Approach. Business Media Praha, Praha.

Machon, V., Levorova, J., Hirjak, D., Drahos, M., Foltan, R. (2017) Temporomandibular joint disc perforation: a retrospective study. Int. J. Oral Maxillofac. Surg. 46(11), 1411-1416.

Mercuri, L. G. (2008) Osteoarthritis, osteoarthrosis, and idiopathic condylar resorption. Oral Maxillofac. Surg. Clin. North Am. 20(2), 169-183.

Miloro, M., Henriksen, B. (2010) Discectomy as the primary surgical option for internal derangement of the temporomandibular joint. J. Oral Maxillofac. Surg. 68(4), 782-789.

Miloro, M., McKnight, M., Han, M. D., Markiewicz, M. R. (2017) Discectomy without replacement improves function in patients with internal derangement of the temporomandibular joint. J. Craniomaxillofac. Surg. 45(9), 1425-1431.

Muñoz-Guerra, M. F., Rodríguez-Campo, F. J., Fernández-Domínguez, M. (2018) The auricular cartilage graft used as interpositional material for disc replacement after failed TMJ operative arthroscopy. J. Stomatol. Oral Maxillofac. Surg. 119(4), 328-336.

Murphy, J. B. (1914) Arthroplasty for intra-articular bony and fibrous ankylosis of the temporomandibular articulation. Report of nine cases. JAMA LXII(23), 1783-1789.

Peer, L. A. (1950) Loss of weight and volume inhuman fat grafts. Plast. Reconstr. Surg. 5, 217-230.

Rattan, V. (2006) A simple technique for use of buccal pad of fat in temporomandibular joint reconstruction. J. Oral Maxillofac. Surg. 64, 1447-1451.

Shen, P., Sun, Q., Xu, W., Zhen, J., Zhang, S., Yang, C. (2015) The fate of autogenous free fat grafts in the human temporomandibular joint using magnetic resonance imaging. J. Craniomaxillofac. Surg. 43(9), 1804-1808.

Silver, C. M. (1984) Long-term results of menisectomy of the temporomandibular joint. Cranio $\mathbf{3}$, 46-57.

Tekin, U., Keller, E. E., DeLone, D. R. (2014) Is autogenous abdominal fat transplantation into a large temporomandibular joint defect following removal of failed alloplastic prosthesisa definitive treatment? J. Oral Maxillofac. Surg. 72, 868-885.

Wolford, L. M., Karras, S. C. (1997) Autologous fat transplantation around temporomandibular joint total joint prostheses: preliminary treatment outcomes. J. Oral Maxillofac. Surg. 55(3), 245-251. 